RESEARCH PAPER

\title{
Impact of UK policy initiatives on use of medicines to aid smoking cessation
}

\author{
R West, M E DiMarino, J Gitchell, A McNeill
}

Tobacco Control 2005;14:166-171. doi: 10.1136/tc.2004.008649

See end of article for authors' affiliations .....

Correspondence to: Professor Robert West, Health Behaviour Unit, Department of

Epidemiology and Public Health, University College London, London WCIE 6BT, UK; robert.wesł@ucl. ac.uk

Received 22 April 2004 Accepted 18 March 2005
Context: Increasing the use of effective smoking cessation aids could in principle have a substantial public health impact. The UK government has undertaken several major policy initiatives to try to increase usage of smoking cessation medicines. It is important to evaluate what effect, if any, these have had to inform future policy in the UK and internationally.

Objective: This study used sales data to examine the impact of government initiatives to increase access to smoking cessation medicines.

Design: Information about prescription and non-prescription sales (1999-2002) was obtained. Estimates of utilisation were compared with findings from the Office of National Statistics (ONS) omnibus surveys. The effects of policy initiatives (making the medicines reimbursable and making them available on general sale outside pharmacies) were assessed by means of time series analysis. In addition a new nicotine replacement therapy (NRT) product (a nicotine lozenge) was launched and the effect of this on total utilisation was assessed.

Results: Making bupropion, and subsequently nicotine replacement therapy (NRT), reimbursable had a major impact in medication usage; the estimated increase in each case was more than 80000 "treatment weeks" purchased per month. In addition, introduction of a nicotine lozenge increased total utilisation and did not detract from usage of other medicines. According to both the sales and the survey data, the proportion of smokers using medicines to aid smoking cessation more than doubled from $8-9 \%$ in 1999 to $17 \%$ in 2002 . The ONS surveys showed no increase in the proportions of smokers making quit attempts and so the effects were solely on the proportions of quit attempts that were aided by medication.

Conclusions: In the UK, making smoking cessation medicines reimbursable led to a large increase in utilisation. While the effect on smoking prevalence would be too small to be detected in national surveys it could have a substantial public health impact.
$\mathrm{F}$ or smokers of 20 cigarettes per day, stopping before age 35 increases life expectancy by eight years and stopping between 45 and 55 years by four years. ${ }^{1}$ For all smokers it reduces the burden of chronic ill health that they carry throughout their lives. Stopping smoking is extremely difficult, however. The chances of long term success of an unaided quit attempt are less than $5 \%{ }^{2}$ This arises at least in part from the effect that nicotine from cigarettes has on the brain. Essentially, it rewards smoking and punishes abstinence leading to a deeply entrenched behaviour pattern that is resistant to conscious attempts to control it. ${ }^{3}$ There is now a consensus that nicotine dependence should be regarded as a chronic, life threatening, psychological disorder. ${ }^{4}$

Both psychological and pharmacological treatments can be effective. Multi-session psychological support from a smoking cessation counsellor, either in groups or one-to-one, can boost long term (>12 month) success rates by about $7 \% .^{5}$ Bupropion and nicotine replacement therapy (NRT) in the form of chewing gum, transdermal patch, inhalator, nasal spray, lozenge, or sublingual tablet can improve long term success rates by a similar amount overall. ${ }^{6}{ }^{7}$ The combination of medication and psychological support can boost long term success rates to approximately $15-20 \%{ }^{8}$ The value for money in terms of gain in life years for a given expenditure is among the highest of all medical treatments. ${ }^{9}$

The USA saw use of NRT increase from $8 \%$ of quit attempts (about 3\% of smokers) in 1986 to $20 \%$ ( $8 \%$ of smokers) in 1996. ${ }^{10}$ A recent US survey indicated that, while there was some reimbursement of smoking cessation treatments, this was far from complete. ${ }^{11}$ Survey data in the UK up until 1997 suggested that about $25 \%$ of quit attempts involved NRTs. ${ }^{12}$
Since 1999, the UK has embarked on an unprecedented effort to expand the utilisation of pharmacotherapy to treat tobacco dependence. It is important to know how successful these efforts have been.

Two strategies have been used (table 1):

- Placing bupropion and all NRT products on National Health Service (NHS) prescription (making them reimbursable)

- Widening sales of NRT products beyond pharmacies to supermarkets and other non-prescription general sales (GSL) settings. (In the UK a distinction is made between pharmacy only over-the-counter sales and general sale.)

This study examined the amount of NRT and bupropion obtained by smokers on a monthly basis in Great Britain from

Table 1 Regulatory and policy changes in the UK concerning smoking cessation medications from 1999

\begin{tabular}{ll}
\hline Regulatory or policy change & Date \\
\hline $\begin{array}{l}\text { Nicotine gum (2 mg) available for general } \\
\text { sale (GSL) }\end{array}$ & 31 March 1999 \\
$\begin{array}{l}\text { Bupropion available on NHS prescription } \\
\text { All smoking cessation products available } \\
\text { on NHS prescription }\end{array}$ & June 2000 \\
$\begin{array}{l}\text { Nicotine gum (4 mg), nicotine lozenge, and } \\
\text { all nicotine patches available for GSL }\end{array}$ & 31 May 2001 \\
\hline
\end{tabular}

NHS prescriptions are free of charge for patients who are under 18 years, over 65 years, on income support, or pregnant; otherwise the charge per prescription was approximately $£ 6$ over the period of study. 
1999 to 2002, the period in which the policy changes were made. It was then possible to make estimates of utilisation and compare these with data from national surveys of smokers. When considering the likely effects of the policy initiatives, one might imagine that making a medication reimbursable on prescription would increase its usage. However, it could also be argued that it would simply transfer the burden of funding from the private individual to the state without increasing usage. It may, for example, lead to a corresponding decrease in over-the-counter sales. Indeed one study found that making smoking cessation medicines reimbursable by a health maintenance organisation in the USA did not lead to an increase in overall usage. ${ }^{13}$ By contrast, an analysis of national data from the USA showed that increasing availability of NRT through a switch to overthe-counter and introduction of new products was associated with large increases in total medication assisted quit attempts. ${ }^{14}$ These two sets of data might suggest that the greatest increase in utilisation comes from widening the range of retail outlets rather than through reimbursement, but it probably depends on the context in which the changes occur so what would happen in the UK is very much an empirical question.

During the study period, a new nicotine lozenge was introduced. It was of interest to know whether this would increase total usage or just substitute for other products. The US study referred to earlier ${ }^{14}$ found an increase when the patch was introduced in 1992, but that was in the context of a relatively immature market. When the lozenge was introduced in the UK the market already had five different forms of NRT, so introduction of a new product may not have boosted total sales.

\section{METHODS}

\section{Data sources}

Information about the prescription and non-prescription sales of smoking cessation products from 1999 to 2002 was obtained from Scriptcount Plus (Taylor Nelson Sofres, London, UK) and InfoScan (Information Resources, Bracknell, UK) respectively.

Scriptcount Plus uses a panel of 875 retail pharmacies to measure the number of prescriptions dispensed, via the patient medication record (PMR) electronic database. The panel is a nationally representative sample based on region and pharmacy size. The total number of prescriptions dispensed across Great Britain is estimated based on a two stage weighting projection and validated against other prescription tracking methods (Prescription Pricing Authority and TNS transaction sales). We obtained the data on the estimated number of prescriptions filled for bupropion and all NRT products (nicotine patch, gum, lozenge, inhaler, microtab, and nasal spray).

Data from InfoScan was used to estimate the nonprescription sales of NRT products to consumers. InfoScan is used to monitor and evaluate product performance in the grocery, health and beauty, and impulse markets. InfoScan tracks retail sales, using data from electronic point of sale (EpoS) scanners and manual store audits. For major markets (for example, supermarket chains), all sales are electronically tracked by EpoS scanning at checkout. For other markets (for example, independent grocers and co-ops) and drug stores (for example, chains and independents), a representative sample of stores is monitored for sales using manual audits or EpoS scanning (when available) and total sales are projected based on the census of all retailers. Information Resources produces estimates of total national sales by combining total sales from major markets and projected total sales from other markets. Coverage of data from InfoScan represents approximately $95 \%$ of all sales in the smoking cessation market. We obtained tabulations of estimated sales as four week blocks of individual products by brand name, product type (patch, gum, etc), and unit counts (pieces per unit).

To obtain information on numbers of quit attempts and to compare estimates of usage from the prescription/purchase data with direct estimates from surveys, we used data from the November and December 1999 and 2002 Office of National Statistics Omnibus surveys. These are household surveys of representative samples of adults in Great Britain. In each survey, a random probability sample of 3000 households is selected using the postcode address file as a sampling frame. One adult aged 16 or over is selected from each household. Further details of the methods are given in the main reports from those surveys. ${ }^{15} 16$

\section{Procedures}

Data were available for number of prescriptions filled per calendar month. The number of units (a "unit" being a unit of sale-for example, a box of nicotine patches) corresponding to a single prescription varies across general practitioners, but data from IMS Health who provide data on prescribing patterns show that the average prescription is for two weeks' supply of medication so that figure was used in this analysis.

Data for the non-prescription sales of NRT products were only available as tabulations in four week blocks (not as calendar months), so we converted these four week blocks into calendar months to be consistent with prescription sales data.

In order to compare data across products with units that contained different numbers of doses we standardised sales data into "treatment weeks". For example, a nicotine patch product that contained a unit count of 14 patches, with a daily application of a new patch, would represent two treatment weeks. Table 2 presents the weekly usage we used to produce a single treatment week for each product type. This was based on recommended doses and does not necessarily represent actual usage which for many of the products is typically lower. For example, actual weekly usage of $2 \mathrm{mg}$ and $4 \mathrm{mg}$ gum is about 50 pieces and actual usage of inhaler is about 25 cartridges. ${ }^{17}$ These data therefore represent "treatment weeks sold".

We estimated usage based on the assumption that smokers obtain an average of four weeks' supply of NRT when making a quit attempt, ${ }^{17}$ so treatment weeks were divided by four to arrive at treatment episodes.

Numbers of quit attempts from the Office of National Statistics (ONS) surveys were calculated by combining smokers who reported having made a quit attempt in the past 12 months with ex-smokers who reported having stopped in the same period. Medication usage was estimated by calculating the percentage of the above smokers/exsmokers who reported purchasing medication or receiving it on prescription. Note that the figures were calculated from the original data because those in the published tables did

Table 2 Manufacturer's recommended daily and weekly usage by product type

\begin{tabular}{lll}
\hline Product type & $\begin{array}{l}\text { Recommended } \\
\text { daily usage }\end{array}$ & $\begin{array}{l}\text { Recommended } \\
\text { weekly usage }\end{array}$ \\
\hline Bupropion & 2 pills & 15 pills \\
Nicotine patch & 1 patch & 7 patches \\
Nicotine gum & 10 pieces & 72 pieces \\
Nicotine lozenge & 10 pieces & 72 pieces \\
Nicotine microtabs & 10 pieces & 72 pieces \\
Nicotine inhaler & 6 cartridges & 42 cartridges \\
Nicotine nasal spray & 16 doses & 100 doses \\
\hline
\end{tabular}


not correspond to the base samples needed (that is, smokers making a quit attempt in the past 12 months).

\section{Analysis}

The time series data were subjected to seasonal decomposition to remove seasonal effects. This was performed on the monthly data. The extent of autocorrelation in the derived series was then assessed. Policy initiatives were added to the model with each marked by setting months before it as 0 and after it as 1. In addition a new lozenge was launched towards the end of 2001 and this event was coded in the same way. A linear regression was undertaken with these and year entered together as predictor variables controlling for any autocorrelation. ${ }^{18}$ The primary outcome measure chosen was total number of treatment weeks because it is the effect on total treatment utilisation that is of greatest interest. The question is: does making NRT or bupropion reimbursable on the NHS actually increase treatment utilisation or merely substitute NHS prescription usage for over-the-counter (OTC) usage? However, we also examined the effects of interventions separately on prescription NRT and OTC NRT. Bupropion was not included separately as a dependent variable because it was made reimbursable on NHS prescription at the same time as it became available, six months into the period for analysis, and starting from a zero base for the first six months of the study period it could only go up at the point the drug was introduced. Analyses were undertaken using SPSS version 11.5. The seasonal decomposition was undertaken using the SEASON command and the main analyses including the determination of and controlling for the autocorrelation function were undertaken using the ARIMA command.

\section{RESULTS}

There was a dramatic increase in sales of medications to aid smoking cessation over the study period, superimposed on strong seasonal fluctuation (fig 1 ). It was found that there was a significant autocorrelation between successive months ( $\mathrm{B}=0.43, \mathrm{p}=005$ ), but no other autoregressive parameters were significant so only this one was used in the model. Table 3 shows the final models for total medication sales and for prescription and OTC NRT sales. Significant effects of making bupropion and NRT reimbursable were identified, controlling for all other variables. Widening general sales access was associated with a non-significant increase in sales. There was a significant increase in total sales following the lozenge launch. Table 3 also confirms that making NRT reimbursable on NHS prescription increased prescription sales (as one would expect) but without any evidence of a decline in OTC sales. The launch of the nicotine lozenge appears to have primarily increased prescription sales.

Figure 2 shows that nicotine gum and patch both accounted for significant OTC purchases of NRT with modest contributions from other products. It is also apparent that OTC patch sales fluctuated with season to a much greater extent than did OTC gum sales.

Figure 3 shows the large rise in nicotine patch prescriptions when NRT became available on NHS prescription. There was only a very small increase in nicotine gum and other products. When the lozenge became available this was taken up more than other non-patch products without diminishing prescriptions of other NRT products. There was a large peak in the early part on 2002. This was the first period of January to March after putting NRT on prescription and appears to reflect the seasonal peak in the context of this change in policy.

Table 4 shows the estimated usage of medication in quit attempts compared with data extracted from the corresponding ONS Omnibus surveys for those years. The usage data estimate from the sales figures correspond closely to those from the ONS surveys. According to the ONS surveys the proportions of smokers making a quit attempt did not change but the proportions using medication more than doubled.

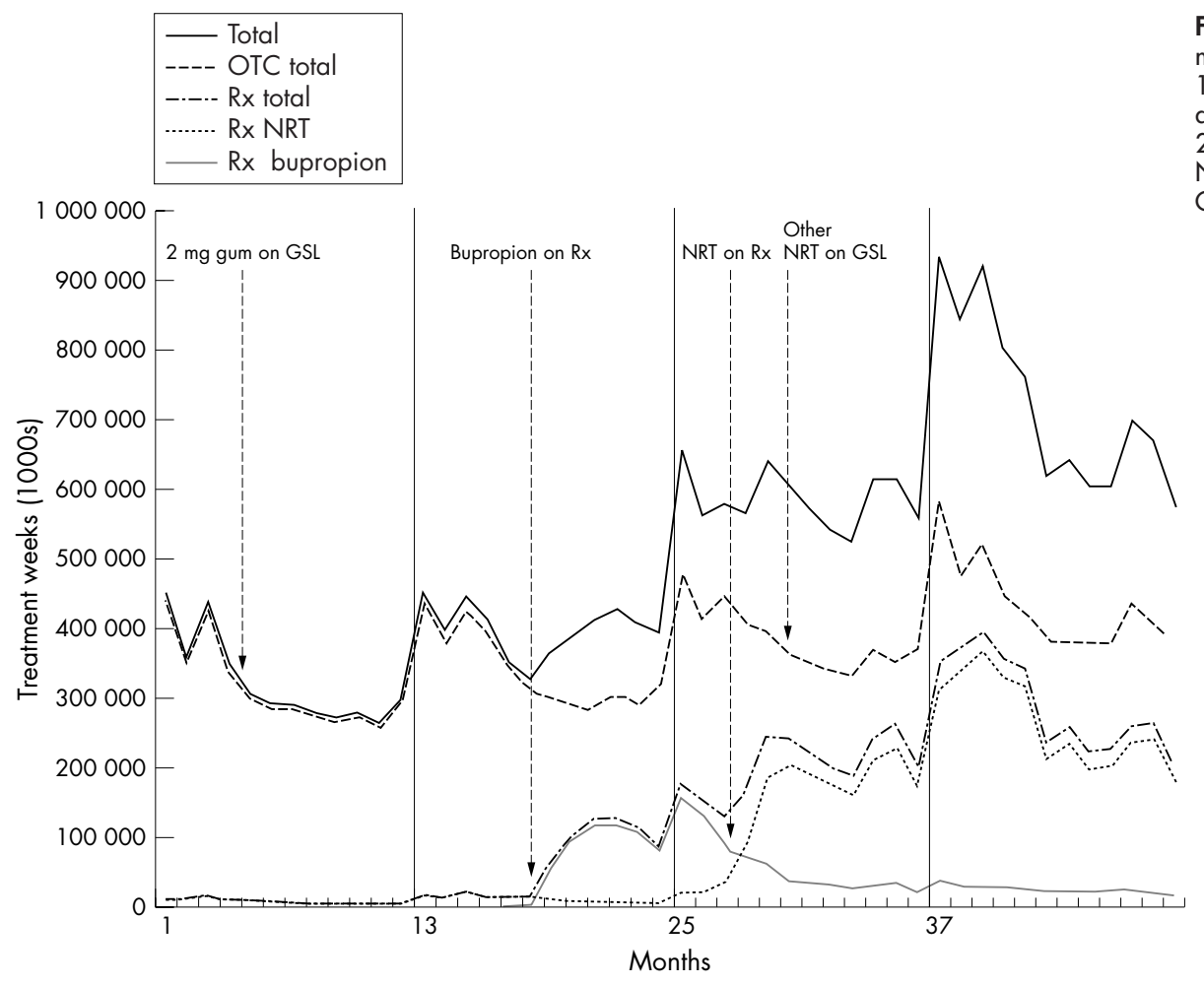

Figure 1 Monthly rates of sales of medications to aid smoking cessation: 1999-2002 (months 1, 13, 25, and 37 are January 1999, 2000, 2001 , and 2002). GSL, general sales; NRT, nicotine replacement therapy; OTC, over-the-counter. 


\begin{tabular}{|c|c|c|c|c|}
\hline & B & SE & T ratio & $\mathrm{p}$ Value \\
\hline \multicolumn{5}{|l|}{ Total } \\
\hline Year & 28504.5 & 16508.1 & 1.73 & 0.09 \\
\hline 2 mg gum on $\mathrm{GSL}^{*}$ & 9733.1 & 29994.3 & 0.32 & 0.75 \\
\hline Bupropion reimbursed & 101629.3 & 25914.8 & 3.92 & 0.0003 \\
\hline NRT reimbursed & 80800.9 & 34188.0 & 2.36 & 0.02 \\
\hline Other NRT on GSL & 55031.5 & 35536.8 & 1.55 & 0.13 \\
\hline Lozenge available & 72621.8 & 29252.2 & 2.48 & 0.02 \\
\hline \multicolumn{5}{|c|}{ Prescription nicotine replacement } \\
\hline Year & -8078.1 & 15372.6 & -0.53 & 0.60 \\
\hline $2 \mathrm{mg}$ gum on $\mathrm{GSL}^{*}$ & 2406.6 & 27897.2 & 0.09 & 0.93 \\
\hline Bupropion reimbursed & 14891.4 & 24037.7 & 0.62 & 0.54 \\
\hline NRT reimbursed & 99343.8 & 31982.9 & 3.11 & 0.003 \\
\hline Other NRT on GSL & 81004.0 & 33296.5 & 2.43 & 0.20 \\
\hline Lozenge available & 100575.1 & 27205.7 & 3.69 & 0.0007 \\
\hline \multicolumn{5}{|c|}{ OTC nicotine replacement } \\
\hline Year & 18452.2 & 7234.06 & 2.55 & 0.014 \\
\hline 2 mg gum on GSL* & -18351.4 & 13315.1 & -1.38 & 0.18 \\
\hline Bupropion reimbursed & 8906.1 & 11783.0 & 0.76 & 0.45 \\
\hline NRT reimbursed & 19005.0 & 14493.6 & 1.31 & 0.20 \\
\hline Other NRT on GSL & 14164.7 & 14866.4 & 0.95 & 0.35 \\
\hline Lozenge available & 24831.0 & 12998.16 & 1.91 & 0.06 \\
\hline
\end{tabular}

*The power to estimate this parameter was limited by the fact that there were very few data points before the event.

GSL, general sales; NRT, nicotine replacement therapy; OTC, over-thecounter; $\mathrm{SE}$, standard error

\section{DISCUSSION}

There was a dramatic increase in sales of medications to aid smoking cessation in the UK from 1999 to 2002. By the end of the period, usage was far higher than in any other country. It was possible to detect increases in total sales immediately after making bupropion and subsequently NRT reimbursable. Sales of bupropion declined sharply at around the time of
NRT becoming reimbursable and it is possible that the rise in NRT prescriptions caused the decline. However, at that time there was also a major tabloid press campaign against bupropion, claiming that it had been responsible for a number of deaths. ${ }^{19}$ There is no way of knowing what would have happened to bupropion sales in the absence of this campaign but it may have limited the overall growth in prescriptions for smoking cessation medications. There was no evidence that putting the medications on prescription reduced OTC purchases. This may be because there was continued advertising direct to smokers.

The experience of the UK appears to be somewhat different from the USA in that the major impact appears to have been the introduction of reimbursement, though in the case of bupropion this was accompanied by launch of a major new drug. Interestingly, this showed no evidence at all of decreasing the OTC market. There are too many uncontrolled variables to be confident about where the source of the difference between the countries lies but the fact that reimbursement was a national policy initiative in UK rather than something initiated by a health insurance organisation may have played a role. It is worth noting, however, that the overall utilisation in the UK is approximately double that of the USA and the UK was on an upward trajectory at the point where the data series ended.

Apart from policy initiatives, there was evidence that launch of a new product might increase usage. The introduction of the lozenge in 2002 did not produce a compensatory reduction in use of other products and therefore contributed to growth in usage overall. It is interesting that the effect was mostly on prescription sales. This may reflect targeting of marketing on general practitioners.

During the study period, there was an expansion in NHS stop smoking clinics providing both behavioural support and medication. One might imagine that this would have had an impact on medication use. However, even in 2002 only $2 \%$ of

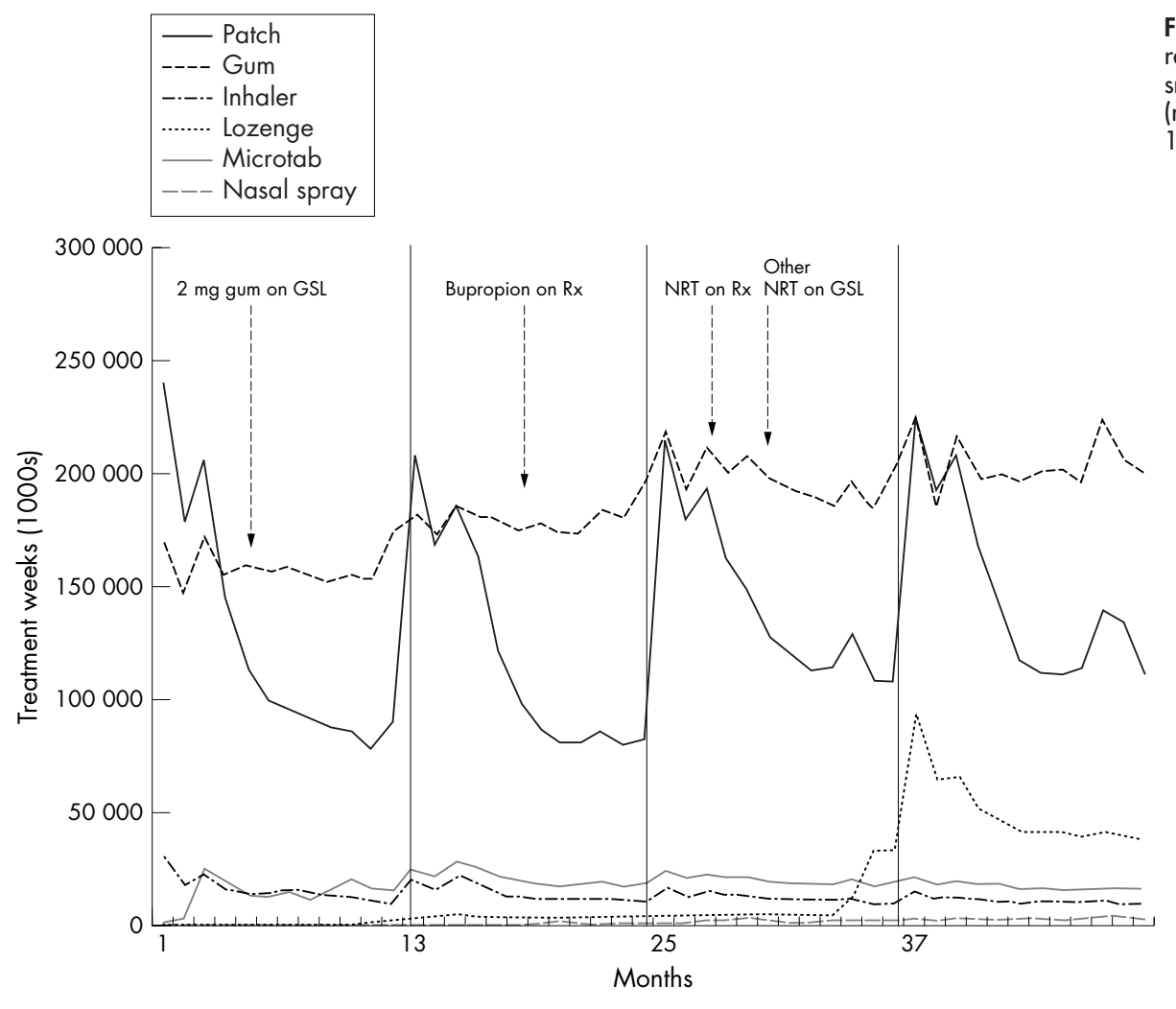

Figure 2 Monthly OTC nicotine replacement therapy purchases to aid smoking cessation: 1999-2002 (months 1, 13, 25, and 37 are January 1999, 2000, 2001, and 2002). 


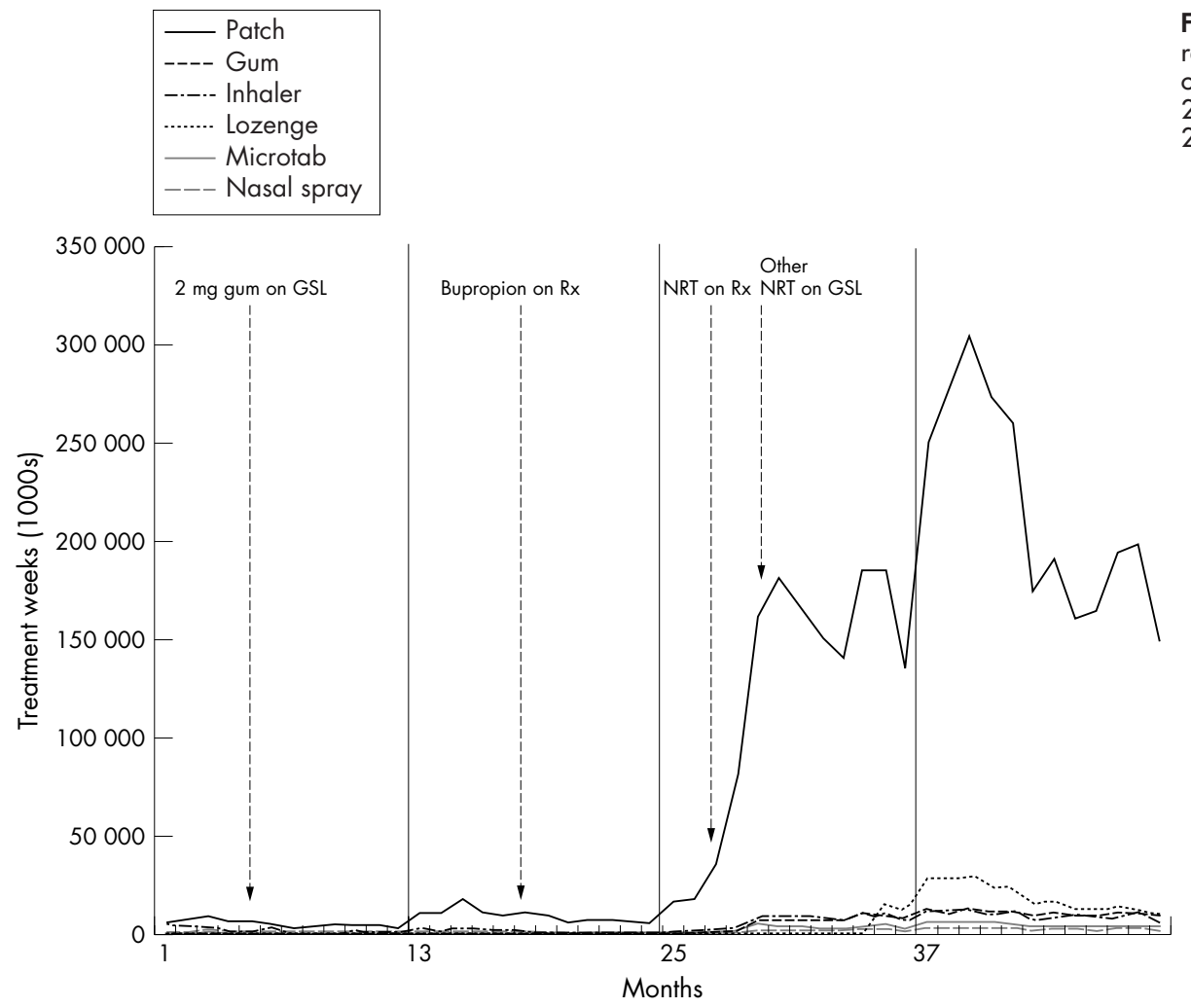

Figure 3 Monthly prescription nicotine replacement therapy use to aid smoking cessation: 1999-2002 (months 1, 13, 25 , and 37 are January 1999, 2000, 2001, and 2002).

smokers were using these services ${ }^{20}$ so the direct effect of the clinics on overall medication usage would have been modest.

The major limitation of this study is the need to make a number of assumptions to go from sales figures to usage rates. However, unless the usage patterns in relation to sales changed substantially over the period under study, the conclusions about changes in usage and the impact of the policy initiatives remain valid. Moreover, where comparisons were possible with the ONS survey data, similar results were obtained.

In principle it is possible that some of the increase in medication usage has arisen from smokers using it to help them reduce their smoking or to ease withdrawal symptoms during short periods of enforced abstinence. However, this is not something that would account for the sharp increases observed in response to policy changes because these were primarily in prescription sales. Moreover, the usage figures derived from the sales data match closely the figures for medication use for cessation found from the national surveys (table 4) which leaves only marginal scope for additional usage for non-cessation purposes. Another potential issue is the role of confounding factors that were not included in the models. In principle these might include changes in the price of NRT or cigarettes, and demographic changes. In practice, over this short time period changes in these variables were minimal.

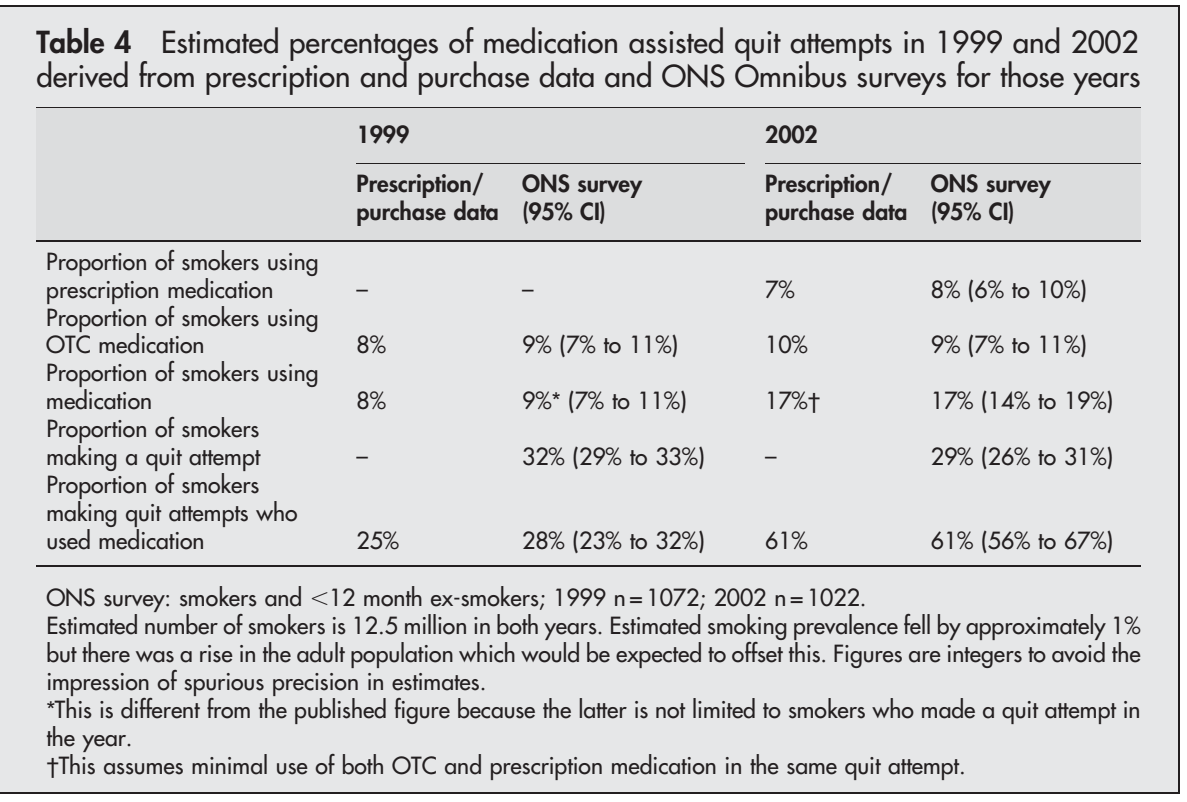




\section{What this paper adds}

This paper shows that in the UK context, with a National Health Service, reimbursement of smoking cessation medications has played a major role in increasing usage and that, as in the USA, introduction of a new product can also increase total usage. It demonstrates that the introduction of reimbursement need not detract from over-the-counter sales. It provides important information on the use of specific smoking cessation medicines.

The large effect of reimbursement on medication usage found in this study conflicts with the finding mentioned in the introduction that making it reimbursable in a health maintenance organisation had no effect. ${ }^{13}$ However, it appears that in that study there was little awareness among smokers of the change and the paper does not make it clear what steps, if any were taken, to inform smokers. In the UK, the change took place in the context of widely disseminated national guidelines for health professionals and a coordinated strategy for tobacco control publicised by the Department of Health. ${ }^{5}$ Common sense would suggest that smokers and health professionals would need to be aware of policy changes of this kind for them to have a significant impact.

It might be imagined that a doubling in the use of medication in quit attempts over a period of three years would produce a detectable reduction in smoking prevalence. In fact, there is evidence of a reduction in smoking prevalence in the UK of about $0.4 \%$ per year since 1999. ${ }^{21}$ However, increased utilisation of smoking cessation medications could not account for this. It has been estimated from clinical trials that NRT with minimal behavioural support boosts $>6$ month continuous abstinence by $5 \%^{7}$-a figure less than that observed in studies where NRT is compared with placebo in the context of behavioural support. An increase over three years from $25 \%$ to $50 \%$ in usage to support quit attempts would see a decrease of $1.25 \%$ in the number of smokers $15 \%$ of $25 \%$ ), other things being equal. With smokers accounting for $26 \%$ of the adult population this corresponds to a decrease in smoking prevalence of approximately $0.32 \%$ in three years ( $1.25 \%$ of $26 \%$ ), or just over $0.1 \%$ per year. This figure is well within the margin of error of annual surveys aimed at assessing prevalence. On the other hand, it would amount to some 50000 additional ex-smokers per year. Taking into account relapse rates in those who have stopped and quit rates in those who have not, this amounts to a minimum of 100000 life years gained and compares favourably with other important public health measures such as the breast screening programme which in the UK is estimated to save approximately 66000 life years annually. ${ }^{22}$

The estimated public health impact of the increase in medication utilisation has been called into question by a report that NRT use in California was not associated with sustained abstinence. ${ }^{23}$ However, this was based on cross sectional survey data and retrospective reports which must have been subject to some serious biases because the overall quit rate calculated was much too high to be genuine. ${ }^{24}$ Nevertheless, we need prospective data comparing success rates in smokers using NRT in self quit attempts with those not using it, controlling for dependence and other possible confounding factors, before we can be confident of the public health impact of policy interventions aimed at increasing medication use.

In conclusion, making bupropion and then NRT reimbursable on NHS prescription increased the total utilisation of smoking cessation medications and did not merely result in prescription use substituting for OTC use. We also found that introducing the lozenge increased overall utilisation rather then substitution. It is quite possible that different effects would have been observed in different national contexts, but it does indicate the potential effect of these kinds of initiatives as public health measures.

\section{Authors' affiliations}

R West, A McNeill, Health Behaviour Unit, Department of Epidemiology and Public Health, University College London, London, UK

M E DiMarino, J Gitchell, Pinney Associates, Bethesda, Maryland, USA

Competing interests: Robert West has undertaken research and consultancy for manufacturers of stop-smoking products; he has received honoraria, travel funds, and hospitality from these companies. Robert West has applied for a patent for a new nicotine replacement product. Ann McNeill has received travel funds and hospitality from manufacturers of stop-smoking products. Joe Gitchell and Michael DiMarino, through Pinney Associates, undertake consultancy for GSK who manufacture nicotine replacement therapies and bupropion. Joe Gitchell has a financial interest in development of novel nicotine replacement products. This study was originated by Ann McNeill who approached GSK for assistance with it; neither she nor Robert West received payment for their work on it.

Robert West is supported by a grant from Cancer Research UK.

\section{REFERENCES}

1 Taylor DH Jr, Hasselblad V, Henley SJ, et al. Benefits of smoking cessation for longevity. Am J Public Health 2002;92:990-6.

2 Hughes JR, Keely J, Naud S. Shape of the relapse curve and long-term abstinence among untreated smokers. Addiction 2004;99:29-38.

3 Balfour DJ. Neuroplasticity within the mesoaccumbens dopamine system and its role in tobacco dependence. Curr Drug Target CNS Neurol Disord 2002;1:413-21.

4 Royal College of Physicians. Nicotine addiction in Britain. London: RCP, 2000.

5 West R, McNeill A, Raw M. Smoking cessation guidelines for health professionals: an update. Health Education Authority. Thorax 2000;55:987-99.

6 West R. Bupropion SR for smoking cessation. Expert Opin Pharmacother 2003;4:533-40.

7 Silagy C, Lancaster T, Stead L, et al. Nicotine replacement therapy for smoking cessation. Cochrane Database Syst Rev 2002:CD000146.

8 West R, McNeill A, Raw M. National smoking cessation guidelines for health professionals: an update. Thorax 2000;55:987-99.

9 National Institute for Clinical Excellence. Nicotine replacement therapy (NRT) and bupropion for smoking cessation. National Institute for Clinical Excellence Technology Appraisal Guidance No. 38. London: NICE, 2002

10 Zhu S, Melcer T, Sun J, et al. Smoking cessation with and without assistance: a population-based analysis. Public health benefit of over-the-counter nicotine medications. Am J Prev Med 2000;18:305-11.

11 Anon. State medicaid coverage for tobacco-dependence treatments - United States, 1994-2002. MMWR Morb Mortal Wkly Rep 2004;53:54-7.

12 West R, McEwen A, Bolling K, et al. Smoking cessation and smoking patterns in the general population: a 1-year follow-up. Addiction 2001;96:891-902.

13 Boyle RG, Solberg LI, Magnan S, et al. Does insurance coverage for drug therapy affect smoking cessation? Health Aff (Millwood) 2002;21(6):162-8.

14 Burton SL, Gitchell JG, Shiffman S. Use of FDA-approved pharmacologic treatments for tobacco dependence - United States, 1984-1998. MMWR Morb Mortal Wkly Rep 2000;49:665-8.

15 Lader D, Meltzer H. Smoking related behaviour and attitudes, 1999. London: Office of National Statistics, 2000.

16 Lader D, Meltzer H. Smoking related attitudes and behaviour, 2002. London: Office of National Statistics, 2003.

17 Hajek P, West R, Foulds J, et al. Randomized comparative trial of nicotine polacrilex, a transdermal patch, nasal spray, and an inhaler. Arch Intern Med 1999;159:2033-8.

18 Box GEP, Jenkins GM. Time series analysis: forecasting and control. New York: Holden-Day, 1976.

19 Ferry LH. The power of the press in smokers' attempts to quit. BM 2002;324:1346-7.

20 Government Statistical Service. Statistics on smoking cessation services in England, April 2001 to March 2002. London: Department of Health, 2003.

21 Jarvis MJ. Monitoring cigarette smoking prevalence in Britain in a timely fashion. Addiction 2003;98:1569-74.

22 Boer R, de Koning $\mathrm{H}$, Threlfall A, et al. Cost effectiveness of shortening screening interval or extending age range of NHS breast screening programme: computer simulation study. BMJ 1998;317:376-9.

23 Pierce JP, Gilpin EA. Impact of over-the-counter sales on effectiveness of pharmaceutical aids for smoking cessation. JAMA 2002;288:1260-4.

24 Stead LF, Davis RM, Fiore MC, et al. Effectiveness of over-the-counter nicotine replacement therapy. JAMA 2002;288:3109-10; author reply, 3110. 